\section{Edoxaban versus Warfarin in Patients with Atrial Fibrillation}

\author{
Robert P. Giugliano, M.D., Christian T. Ruff, M.D., M.P.H., Eugene Braunwald, M.D., \\ Sabina A. Murphy, M.P.H., Stephen D. Wiviott, M.D., Jonathan L. Halperin, M.D., \\ Albert L. Waldo, M.D., Michael D. Ezekowitz, M.D., D.Phil., Jeffrey I. Weitz, M.D., \\ Jindřich Špinar, M.D., Witold Ruzyllo, M.D., Mikhail Ruda, M.D., \\ Yukihiro Koretsune, M.D., Joshua Betcher, Ph.D., Minggao Shi, Ph.D., \\ Laura T. Grip, A.B., Shirali P. Patel, B.S., Indravadan Patel, M.D., \\ James J. Hanyok, Pharm.D., Michele Mercuri, M.D., and Elliott M. Antman, M.D., \\ for the ENGAGE AF-TIMI 48 Investigators*
}

ABSTRACT

\section{BACKGROUND}

Edoxaban is a direct oral factor Xa inhibitor with proven antithrombotic effects. The long-term efficacy and safety of edoxaban as compared with warfarin in patients with atrial fibrillation is not known.

\section{METHODS}

We conducted a randomized, double-blind, double-dummy trial comparing two once-daily regimens of edoxaban with warfarin in 21,105 patients with moderateto-high-risk atrial fibrillation (median follow-up, 2.8 years). The primary efficacy end point was stroke or systemic embolism. Each edoxaban regimen was tested for noninferiority to warfarin during the treatment period. The principal safety end point was major bleeding.

\section{RESULTS}

The annualized rate of the primary end point during treatment was $1.50 \%$ with warfarin (median time in the therapeutic range, 68.4\%), as compared with $1.18 \%$ with high-dose edoxaban (hazard ratio, 0.79; 97.5\% confidence interval [CI], 0.63 to $0.99 ; \mathrm{P}<0.001$ for noninferiority) and $1.61 \%$ with low-dose edoxaban (hazard ratio, $1.07 ; 97.5 \% \mathrm{CI}, 0.87$ to $1.31 ; \mathrm{P}=0.005$ for noninferiority). In the intention-to-treat analysis, there was a trend favoring high-dose edoxaban versus warfarin (hazard ratio, $0.87 ; 97.5 \% \mathrm{CI}, 0.73$ to $1.04 ; \mathrm{P}=0.08$ ) and an unfavorable trend with low-dose edoxaban versus warfarin (hazard ratio, $1.13 ; 97.5 \% \mathrm{CI}, 0.96$ to $1.34 ; \mathrm{P}=0.10$ ). The annualized rate of major bleeding was $3.43 \%$ with warfarin versus $2.75 \%$ with highdose edoxaban (hazard ratio, $0.80 ; 95 \% \mathrm{CI}, 0.71$ to 0.91 ; $\mathrm{P}<0.001$ ) and $1.61 \%$ with low-dose edoxaban (hazard ratio, 0.47 ; $95 \% \mathrm{CI}, 0.41$ to 0.55 ; $\mathrm{P}<0.001$ ). The corresponding annualized rates of death from cardiovascular causes were $3.17 \%$ versus 2.74\% (hazard ratio, $0.86 ; 95 \% \mathrm{CI}, 0.77$ to $0.97 ; \mathrm{P}=0.01$ ), and $2.71 \%$ (hazard ratio, 0.85 ; $95 \% \mathrm{CI}, 0.76$ to $0.96 ; \mathrm{P}=0.008$ ), and the corresponding rates of the key secondary end point (a composite of stroke, systemic embolism, or death from cardiovascular causes) were $4.43 \%$ versus $3.85 \%$ (hazard ratio, 0.87 ; $95 \% \mathrm{CI}, 0.78$ to 0.96 ; $\mathrm{P}=0.005$ ), and $4.23 \%$ (hazard ratio, $0.95 ; 95 \% \mathrm{CI}, 0.86$ to $1.05 ; \mathrm{P}=0.32$ ).

\section{CONCLUSIONS}

Both once-daily regimens of edoxaban were noninferior to warfarin with respect to the prevention of stroke or systemic embolism and were associated with significantly lower rates of bleeding and death from cardiovascular causes. (Funded by Daiichi Sankyo Pharma Development; ENGAGE AF-TIMI 48 ClinicalTrials.gov number, NCT00781391.)
From Brigham and Women's Hospital and Harvard Medical School, Boston (R.P.G., C.T.R., E.B., S.A.M., S.D.W., L.T.G., E.M.A.); Mount Sinai Medical Center, New York (J.L.H.); University Hospitals Case Medical Center, Cleveland (A.L.W.); Thomas Jefferson Medical College, Philadelphia (M.D.E.); McMaster University, Hamilton, ON, Canada (J.I.W.); University Hospital, Jihlavska, Brno, Czech Republic (J.S.); Institute of Cardiology, Warsaw, Poland (W.R.); Cardiology Research Center, Moscow (M.R.); National Hospital Organization, Osaka National Hospital, Osaka, Japan (Y.K.); Quintiles, Durham, NC (J.B., S.P.P.); and Daiichi Sankyo Pharma Development, Edison, NJ (M.S., I.P., J.J.H., M.M.). Address reprint requests to Dr. Giugliano at the Division of Cardiovascular Medicine, Brigham and Women's Hospital, TIMI Study Group, 350 Longwood Ave., 1st. Flr., Boston, MA 02115, or at rgiugliano@partners.org.

Drs. Giugliano and Ruff contributed equally to this article.

* Members of the Effective Anticoagulation with Factor Xa Next Generation in Atrial Fibrillation-Thrombolysis in Myocardial Infarction 48 (ENGAGE AF-TIMI 48) team are listed in the Supplementary Appendix, available at NEJM.org.

This article was published on November 19 , 2013, at NEJM.org.

N Engl J Med 2013;369:2093-104. DOI: 10.1056/NEJMoal310907

Copyright @) 2013 Massachusetts Medical Society 
$\mathrm{E}$

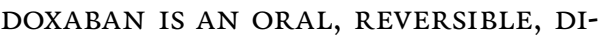
rect factor Xa inhibitor with a linear and predictable pharmacokinetic profile and $62 \%$ oral bioavailability. ${ }^{1}$ It achieves maximum concentrations within 1 to 2 hours, and $50 \%$ is excreted by the kidney. ${ }^{2}$ A randomized, doseranging, warfarin-controlled, phase 2 study involving 1146 patients with atrial fibrillation showed that once-daily doses of edoxaban $(60 \mathrm{mg}$ or $30 \mathrm{mg}$ ) were safer than twice-daily doses. ${ }^{3}$ Pharmacokinetic modeling and simulation showed that patients with low body weight, moderate-tosevere renal dysfunction, or concomitant use of a potent P-glycoprotein inhibitor should have the edoxaban dose reduced by $50 \% .{ }^{4} \mathrm{~A}$ phase 3 study involving 8292 patients with acute venous thromboembolism showed that once-daily edoxaban at a dose of $60 \mathrm{mg}$ (reduced to $30 \mathrm{mg}$ in selected patients) was as effective as warfarin for the prevention of recurrent symptomatic venous thromboembolism and was associated with a significantly lower rate of bleeding. ${ }^{5}$ We compared two dose regimens of once-daily edoxaban with warfarin in patients with atrial fibrillation who were at moderate-to-high risk for stroke.

\section{METHODS}

\section{STUDY OVERSIGHT}

The trial was designed and led by an executive committee, in coordination with an international steering committee (see the Supplementary Appendix, available with the full text of this article at NEJM.org). The protocol and amendments were approved by the ethics committee at each participating center. All the patients provided written informed consent. An independent data and safety monitoring committee performed multiple safety reviews. The Thrombolysis in Myocardial Infarction Study Group coordinated the trial and performed all the analyses independently using raw data. Quintiles, a contract research organization, managed the database and monitored the study sites. All the authors participated in the design of the trial and in the analysis of the data. The first author wrote the first draft of the manuscript, and all the authors participated in subsequent drafts and made the decision to submit the manuscript for publication. All the authors vouch for the accuracy and completeness of the data and the fidelity of the study to the protocol, which is available at NEJM.org. There were no contractual agreements with the sponsor that could have denied the investigators the right to examine the data independently or submit the manuscript for publication without consent of the sponsor.

\section{TRIAL DESIGN}

The Effective Anticoagulation with Factor Xa Next Generation in Atrial Fibrillation-Thrombolysis in Myocardial Infarction 48 (ENGAGE AF-TIMI 48) trial was a three-group, randomized, doubleblind, double-dummy trial comparing two dose regimens of edoxaban with warfarin. We conducted the trial at 1393 centers in 46 countries (see the Supplementary Appendix). Patients were enrolled during the period from November 19, 2008, through November 22, 2010. The protocol and statistical analysis plan have been described previously. $^{6}$

\section{STUDY POPULATION}

Eligible patients were 21 years of age or older and had atrial fibrillation documented by means of an electrical tracing within the 12 months preceding randomization, a score of 2 or higher on the $\mathrm{CHADS}_{2}$ risk assessment, and anticoagulation therapy planned for the duration of the trial. ${ }^{7}$ Scores on the $\mathrm{CHADS}_{2}$ range from 0 to 6 , with higher scores indicating a greater risk of stroke; congestive heart failure, hypertension, diabetes, and an age of 75 years or older are each assigned 1 point, and prior stroke or transient ischemic attack is assigned 2 points. Key exclusion criteria were atrial fibrillation due to a reversible disorder; an estimated creatinine clearance of less than $30 \mathrm{ml}$ per minute; a high risk of bleeding; use of dual antiplatelet therapy; moderate-tosevere mitral stenosis; other indications for anticoagulation therapy; acute coronary syndromes, coronary revascularization, or stroke within 30 days before randomization; and an inability to adhere to study procedures. ${ }^{6}$

\section{RANDOMIZATION AND STUDY DRUGS}

Patients were randomly assigned, in a 1:1:1 ratio, to receive warfarin, dose-adjusted to achieve an international normalized ratio (INR) of 2.0 to 3.0, or to receive high-dose or low-dose edoxaban. Randomization was performed with the use of a central, 24-hour, interactive, computerized response system. Patients who were already taking 
a vitamin $\mathrm{K}$ antagonist underwent randomization after the INR was 2.5 or less. Randomization was stratified according to the following characteristics: $\mathrm{CHADS}_{2}$ score of 2 or 3 versus a score of 4,5 , or 6 and status with respect to the need for a reduction in the edoxaban dose. ${ }^{6}$

The high-dose edoxaban group received $60 \mathrm{mg}$, and the low-dose group $30 \mathrm{mg}$. For patients in either group, the dose was halved if any of the following characteristics were present at the time of randomization or during the study: estimated creatinine clearance of 30 to $50 \mathrm{ml}$ per minute, a body weight of $60 \mathrm{~kg}$ or less, or the concomitant use of verapamil or quinidine (potent P-glycoprotein inhibitors). A protocol amendment on December 22, 2010, mandated similar dose modifications in the case of concomitant use of dronedarone. After randomization, standard dosing was resumed if verapamil, quinidine, or dronedarone was discontinued and if there had been no other reason for a reduction of the edoxaban dose. Each patient received two sets of study drugs: either active edoxaban and a placebo matching warfarin, or a placebo matching edoxaban and active warfarin.

The INR was measured at least monthly with the use of an encrypted point-of-care device. To maintain blinding, sham INR values were generated for patients who were randomly assigned to edoxaban. The time in the therapeutic range in the warfarin group was calculated by means of linear interpolation, ${ }^{8}$ with INR values rounded to the nearest $0.1 .^{9}$ Study visits were scheduled on days $8,15,29$, and 60 , at month 3 , and at least every 3 months thereafter.

At the end of the trial, patients made the transition to open-label oral anticoagulation therapy with the use of a detailed plan (see the study protocol). Patients who made the transition from edoxaban to an open-label vitamin $\mathrm{K}$ antagonist received both active low-dose edoxaban and an open-label vitamin $\mathrm{K}$ antagonist until the INR reached 2.0 or for 2 weeks (whichever came first). At least three INR measurements were mandated between days 4 and 14 of the transition period; the use of an approved dosing algorithm for the vitamin $\mathrm{K}$ antagonist was required.

\section{STUDY END POINTS}

The primary efficacy end point was the time to the first adjudicated stroke (ischemic or hemor- rhagic) or systemic embolic event. The principal safety end point was adjudicated major bleeding during treatment, as defined by the International Society on Thrombosis and Haemostasis. ${ }^{10}$ Key secondary composite end points included the following: stroke, systemic embolic event, or death from cardiovascular causes (including bleeding); myocardial infarction, stroke, systemic embolic event, or death from cardiovascular causes; and stroke, systemic embolic event, or death from any cause. Net clinical end points included composites of stroke, systemic embolic event, major bleeding, or death; disabling stroke, life-threatening bleeding, or death; and stroke, systemic embolic event, life-threatening bleeding, or death.

An independent clinical end-point committee, whose members were unaware of the study assignment, adjudicated all deaths and suspected cerebrovascular events, systemic embolic events, myocardial infarctions, bleeding events, and hepatic events. Details of the definitions used by the clinical end-point committee are provided in the protocol.

\section{STATISTICAL ANALYSIS}

The primary efficacy analysis, which tested whether either dose regimen of edoxaban was noninferior to warfarin, was performed with the use of a Cox proportional-hazards model that included treatment groups and the two randomization stratification factors. This analysis included data from patients who underwent randomization and received at least one dose of the study drug during the treatment period (modified intention-to-treat population). The treatment period was defined as the period between administration of the first dose of the study drug and either 3 days after the receipt of the last dose or the end of the double-blind therapy (whichever came first), with interval censoring of events during study-drug interruptions that lasted more than 3 days. To satisfy noninferiority, the upper boundary of the one-sided $97.5 \%$ confidence interval for the hazard ratio of the primary efficacy end point comparing edoxaban with warfarin could not exceed 1.38, which was an estimate that preserved at least $50 \%$ of the benefit of warfarin over placebo. ${ }^{11,12}$

If an edoxaban dosing regimen met the prespecified criteria for noninferiority, that dose 
Table 1. Demographic and Clinical Characteristics of the Patients.*

\begin{tabular}{|c|c|c|c|}
\hline Characteristic & $\begin{array}{c}\text { Warfarin } \\
(\mathrm{N}=7036)\end{array}$ & $\begin{array}{l}\text { High-Dose } \\
\text { Edoxaban } \\
(\mathrm{N}=7035)\end{array}$ & $\begin{array}{l}\text { Low-Dose } \\
\text { Edoxaban } \\
(\mathrm{N}=7034)\end{array}$ \\
\hline \multicolumn{4}{|l|}{ Age $-y r$} \\
\hline Median & 72 & 72 & 72 \\
\hline Interquartile range & $64-78$ & $64-78$ & $64-78$ \\
\hline Female sex — no. (\%) & $2641(37.5)$ & 2669 (37.9) & $2730(38.8)$ \\
\hline \multicolumn{4}{|l|}{ Region — no. (\%) } \\
\hline North America & $1562(22.2)$ & $1559(22.2)$ & $1560(22.2)$ \\
\hline Latin America & $888(12.6)$ & $886(12.6)$ & $887(12.6)$ \\
\hline Western Europe & $1078(15.3)$ & $1079(15.3)$ & $1079(15.3)$ \\
\hline Eastern Europe & $2381(33.8)$ & $2383(33.9)$ & $2380(33.8)$ \\
\hline Asia-Pacific region and South Africa & $1127(16.0)$ & $1128(16.0)$ & $1128(16.0)$ \\
\hline Paroxysmal atrial fibrillation — no. (\%) & $1778(25.3)$ & $1753(24.9)$ & $1835(26.1)$ \\
\hline \multicolumn{4}{|l|}{ Qualifying risk factor — no. (\%) } \\
\hline Age $\geq 75 \mathrm{yr}$ & $2820(40.1)$ & $2848(40.5)$ & $2806(39.9)$ \\
\hline Prior stroke or transient ischemic attack & $1991(28.3)$ & $1976(28.1)$ & $2006(28.5)$ \\
\hline Congestive heart failure & $4048(57.5)$ & $4097(58.2)$ & $3979(56.6)$ \\
\hline Diabetes mellitus & $2521(35.8)$ & $2559(36.4)$ & $2544(36.2)$ \\
\hline Hypertension requiring treatment & $6588(93.6)$ & $6591(93.7)$ & $6575(93.5)$ \\
\hline $\mathrm{CHADS}_{2}$ score $\dagger$ & $2.8 \pm 1.0$ & $2.8 \pm 1.0$ & $2.8 \pm 1.0$ \\
\hline$\leq 3-$ no. $(\%)$ & $5445(77.4)$ & $5422(77.1)$ & $5470(77.8)$ \\
\hline $4-6-$ no. $(\%)$ & $1591(22.6)$ & $1613(22.9)$ & $1564(22.2)$ \\
\hline Dose reduction at randomization - no. (\%) $\ddagger$ & $1787(25.4)$ & $1784(25.4)$ & $1785(25.4)$ \\
\hline Creatinine clearance $\leq 50 \mathrm{ml} / \mathrm{min}$ & $1361(19.3)$ & $1379(19.6)$ & $1334(19.0)$ \\
\hline Weight $\leq 60 \mathrm{~kg}$ & $701(10.0)$ & $684(9.7)$ & $698(9.9)$ \\
\hline Use of verapamil or quinidine & $243(3.5)$ & $258(3.7)$ & $260(3.7)$ \\
\hline Previous use of vitamin $\mathrm{K}$ antagonist for $\geq 60$ days - no. (\%) & $4138(58.8)$ & $4140(58.8)$ & $4163(59.2)$ \\
\hline \multicolumn{4}{|l|}{ Medication at time of randomization — no. (\%) } \\
\hline Aspirin & $2092(29.7)$ & $2070(29.4)$ & $2018(28.7)$ \\
\hline Thienopyridine & $164(2.3)$ & $174(2.5)$ & $149(2.1)$ \\
\hline Amiodarone & $827(11.8)$ & $866(12.3)$ & $799(11.4)$ \\
\hline Digoxin or digitalis preparation & $2176(30.9)$ & $2078(29.5)$ & $2073(29.5)$ \\
\hline
\end{tabular}

* Plus-minus values are means \pm SD. There were no significant differences between the treatment groups in any of the characteristics listed. $\uparrow$ Scores on the $\mathrm{CHADS}_{2}$ range from 0 to 6 , with higher scores indicating a greater risk of stroke; congestive heart failure, hypertension, diabetes, and an age of 75 years or older are each assigned 1 point, and prior stroke or transient ischemic attack is assigned 2 points.

$\leftarrow$ Patients with a creatinine clearance of $50 \mathrm{ml}$ per minute or less, those with a body weight of $60 \mathrm{~kg}$ or less, and those who were receiving the concomitant potent P-glycoprotein inhibitors verapamil or quinidine at randomization received a dose of edoxaban that was reduced by $50 \%$ to maintain an exposure level similar to that of patients who did not have any of these three factors. Patients may have had more than one reason for dose adjustment.

was then compared with warfarin in a test of superiority with the use of data from the intention-to-treat population, with all primary-endpoint events that occurred during the overall study period (i.e., from randomization to the end of the treatment period) considered in the analysis. To control the overall rate of a type I error with a two-sided alpha significance level of 
0.025 for superiority, sequential multiplicityadjustment procedures (closed testing) were used in a hierarchical fashion to test secondary end points in the intention-to-treat population (Fig. S1 in the Supplementary Appendix). We calculated that with approximately 672 primary-end-point events, the study would have more than $87 \%$ power to reject the null hypothesis that edoxaban was inferior to warfarin. ${ }^{6}$

\section{RESULTS}

\section{PATIENTS AND FOLLOW-UP}

The characteristics of the patients at baseline were well balanced (Table 1). Complete information on the primary end point was ascertained for $99.5 \%$ of the total 56,346 patient-years of potential follow-up (Fig. S2 in the Supplementary Appendix). One patient was lost to follow-up, and 244 patients withdrew consent to follow-up; 182 of these patients had no known primary-endpoint event and were not known to be dead.

\section{STUDY DRUGS}

A total of 21,105 patients underwent randomization, of whom $21,026(99.6 \%)$ received the study drug. A total of 5330 patients (25.3\%) received a reduced dose of edoxaban or matching placebo at randomization, with similar rates in the three treatment groups. After randomization, dose reductions occurred in $7.1 \%$ of the patients, and dose increases in $1.2 \%$, with similar rates in the three treatment groups. The median duration of treatment exposure was 907 days, excluding interruptions; the median follow-up was 1022 days (2.8 years).

Fewer patients in the warfarin group than in either edoxaban group completed the study without drug interruption (2421 in the warfarin group, as compared with 2621 in the high-dose edoxaban group and 2673 in the low-dose edoxaban group; $\mathrm{P}<0.001$ for the comparisons of each dose of edoxaban with warfarin). Premature permanent discontinuation of the study drugs occurred in 2417 patients, 2415 patients, and 2309 patients in the three groups, respectively (Table S1 in the Supplementary Appendix). In the warfarin group, the median time in the therapeutic range was $68.4 \%$ of the treatment period (interquartile range, 56.5 to 77.4 ), and the mean $( \pm S D)$ time in the therapeutic range was
$64.9 \pm 18.7 \%$ of the treatment period; the INR was between 1.8 and 3.2 for $83.1 \%$ of the treatment period.

\section{PRIMARY END POINT}

During the treatment period, a stroke or systemic embolic event occurred in 232 patients in the warfarin group (representing a rate of $1.50 \%$ per year), as compared with 182 patients in the highdose edoxaban group (a rate of $1.18 \%$ per year; hazard ratio vs. warfarin, $0.79 ; 97.5 \%$ confidence interval [CI], 0.63 to 0.99 ; $\mathrm{P}<0.001$ for noninferiority, $\mathrm{P}=0.02$ for superiority) and 253 patients in the low-dose edoxaban group (a rate of $1.61 \%$ per year; hazard ratio vs. warfarin, 1.07; 97.5\% CI, 0.87 to $1.31 ; \mathrm{P}=0.005$ for noninferiority, $\mathrm{P}=0.44$ for superiority) (Table 2).

In the prespecified superiority analysis for efficacy that was performed in the intention-totreat population with data from the overall study period, the annualized rate of the primary end point was $1.80 \%$ in the warfarin group, as compared with $1.57 \%$ in the high-dose edoxaban group (hazard ratio vs. warfarin, 0.87 ; $97.5 \% \mathrm{CI}$, 0.73 to $1.04 ; \mathrm{P}=0.08$ ) and $2.04 \%$ in the low-dose edoxaban group (hazard ratio vs. warfarin, 1.13; 97.5\% CI, 0.96 to 1.34; $\mathrm{P}=0.10$ ) (Table 2 and Fig. 1A). The annualized rate of hemorrhagic stroke was $0.47 \%$ with warfarin, as compared with $0.26 \%$ with high-dose edoxaban (hazard ratio, $0.54 ; 95 \% \mathrm{CI}, 0.38$ to 0.77 ; $\mathrm{P}<0.001$ ) and $0.16 \%$ with low-dose edoxaban (hazard ratio, $0.33 ; 95 \% \mathrm{CI}, 0.22$ to $0.50 ; \mathrm{P}<0.001)$. The rate of ischemic stroke was $1.25 \%$ with warfarin as compared with $1.25 \%$ with high-dose edoxaban (hazard ratio, 1.00; 95\% CI, 0.83 to 1.19; $\mathrm{P}=0.97$ ) and $1.77 \%$ with low-dose edoxaban (hazard ratio, 1.41; 95\% CI, 1.19 to 1.67; $\mathrm{P}<0.001)$.

At the end of the trial, seven primary-endpoint events occurred in each treatment group during the 30-day transition period from treatment with the blinded study drug to receipt of an open-label anticoagulant agent. The rates of major bleeding and death were also similar among the treatment groups during this transition period.

\section{BLEEDING}

The annualized rate of major bleeding events was $3.43 \%$ with warfarin, as compared with $2.75 \%$ with high-dose edoxaban (hazard ratio, 0.80; 95\% 


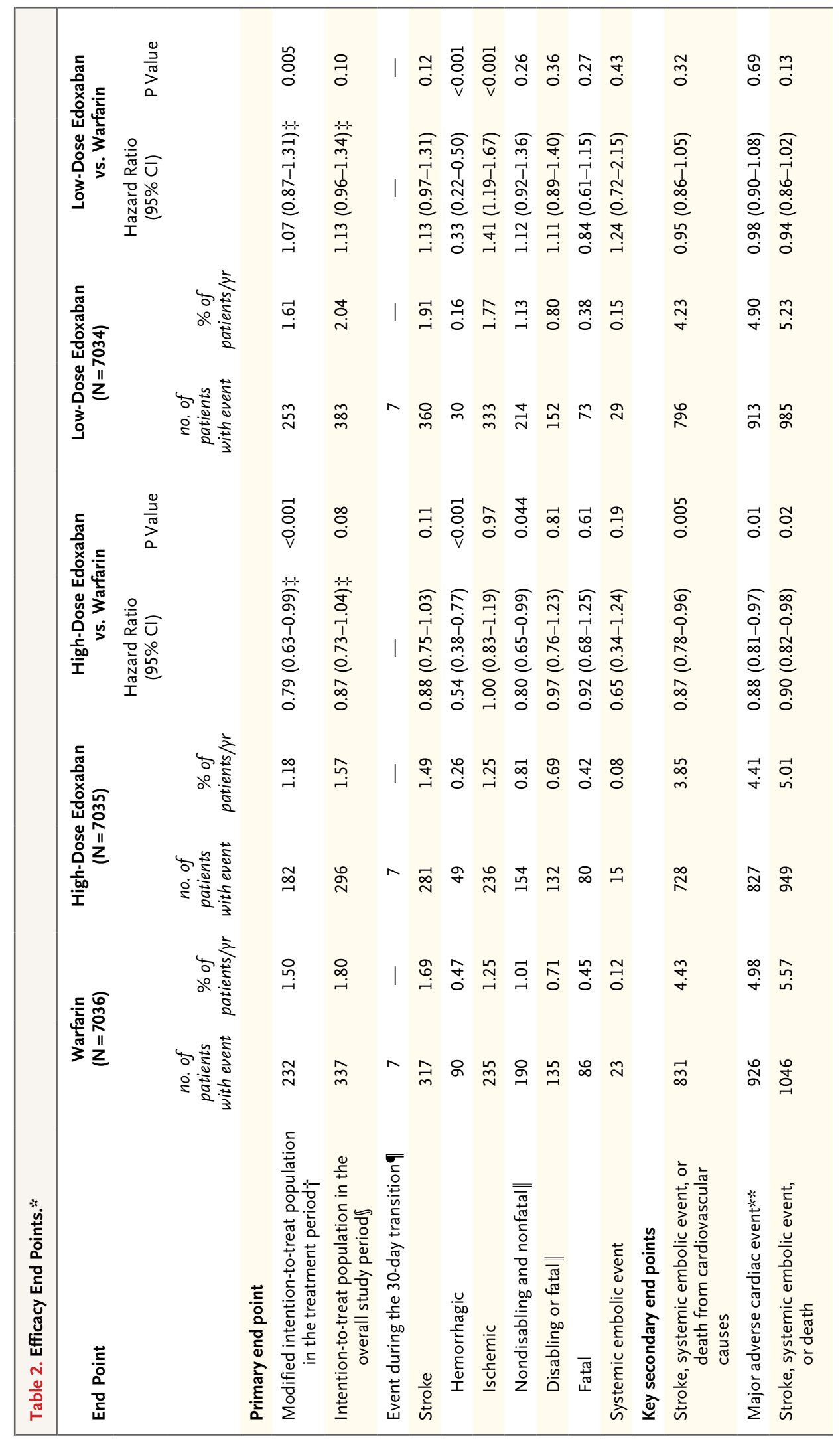




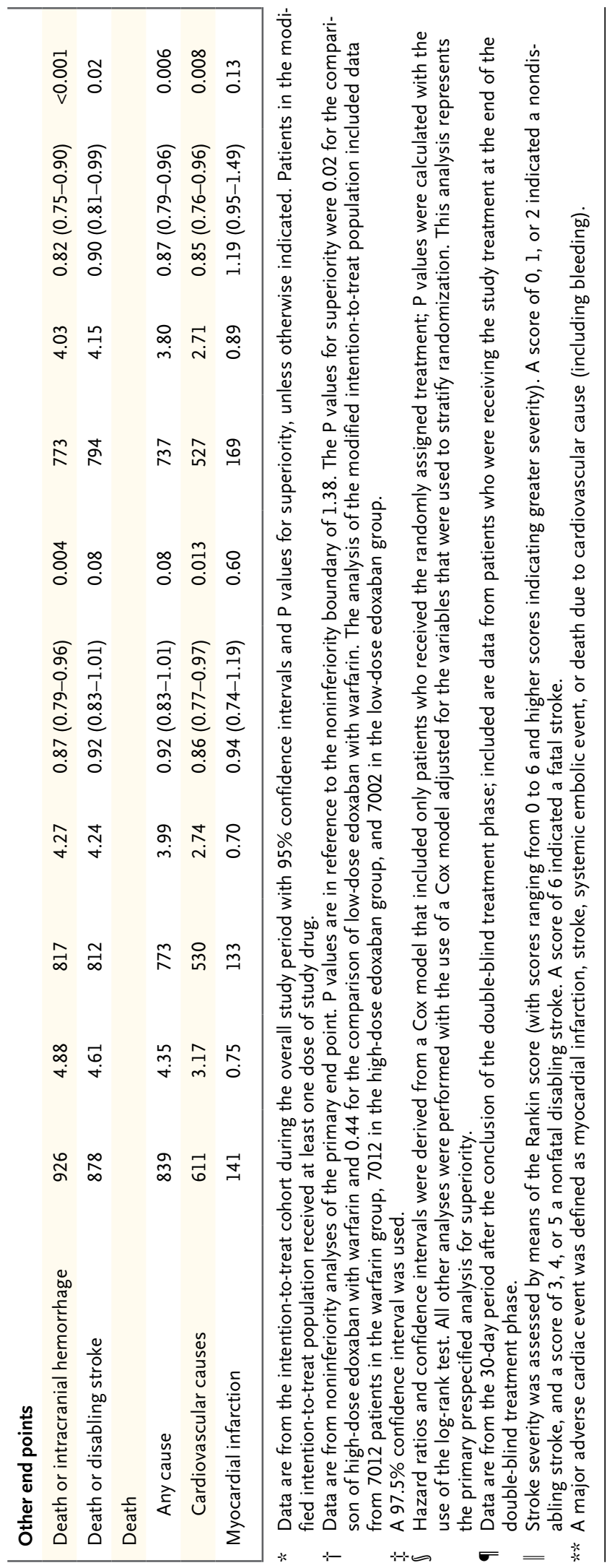

CI, 0.71 to $0.91 ; \mathrm{P}<0.001$ ) and $1.61 \%$ with lowdose edoxaban (hazard ratio, 0.47; 95\% CI, 0.41 to $0.55 ; \mathrm{P}<0.001$ ) (Table 3 and Fig. $1 \mathrm{~B}$ ). The rates of life-threatening bleeding, intracranial bleeding, and major bleeding plus clinically relevant nonmajor bleeding were $0.78 \%, 0.85 \%$, and $13.02 \%$, respectively, with warfarin, as compared with $0.40 \%, 0.39 \%$, and $11.10 \%$, respectively, with high-dose edoxaban and $0.25 \%, 0.26 \%$, and $7.97 \%$, respectively, with low-dose edoxaban $(\mathrm{P}<0.001$ for the comparison of warfarin with each dose of edoxaban). The annualized rate of major gastrointestinal bleeding was higher with high-dose edoxaban than with warfarin $(1.51 \%$ vs. $1.23 \%$ ), but the rate was lowest with low-dose edoxaban $(0.82 \%)$.

\section{SECONDARY AND OTHER EFFICACY OUTCOMES}

The rates of all three prespecified secondary composite outcomes were significantly lower with high-dose edoxaban than with warfarin (Table 2); there were no significant differences between low-dose edoxaban and warfarin in the rates of those outcomes. Treatment with edoxaban was associated with lower annualized rates of death from cardiovascular causes than was warfarin: $3.17 \%$ with warfarin, as compared with $2.74 \%$ with high-dose edoxaban (hazard ratio, 0.86; $95 \% \mathrm{CI}, 0.77$ to $0.97 ; \mathrm{P}=0.01$ ) and $2.71 \%$ with low-dose edoxaban (hazard ratio, $0.85 ; 95 \% \mathrm{CI}$, 0.76 to $0.96 ; \mathrm{P}=0.008$ ), with findings in a similar direction for the rate of death from any cause.

The annualized rate of the primary net clinical outcome (death from any cause, stroke, systemic embolic event, or major bleeding) was significantly lower with both edoxaban regimens than with warfarin: $8.11 \%$ with warfarin, as compared with $7.26 \%$ with high-dose edoxaban (hazard ratio, $0.89 ; 95 \% \mathrm{CI}, 0.83$ to $0.96 ; \mathrm{P}=0.003$ ) and $6.79 \%$ with low dose-edoxaban (hazard ratio, 0.83; 95\% CI, 0.77 to 0.90; $\mathrm{P}<0.001$ ) (Table 3). Similarly, as compared with warfarin, both edoxaban regimens were associated with significantly lower rates of the secondary net clinical outcome of death from any cause, disabling stroke, or lifethreatening bleeding, and the tertiary net clinical outcome of stroke, systemic embolic event, lifethreatening bleeding, or death from any cause.

\section{SUBGROUPS}

In subgroup analyses of the primary efficacy end point, there were significant interactions 


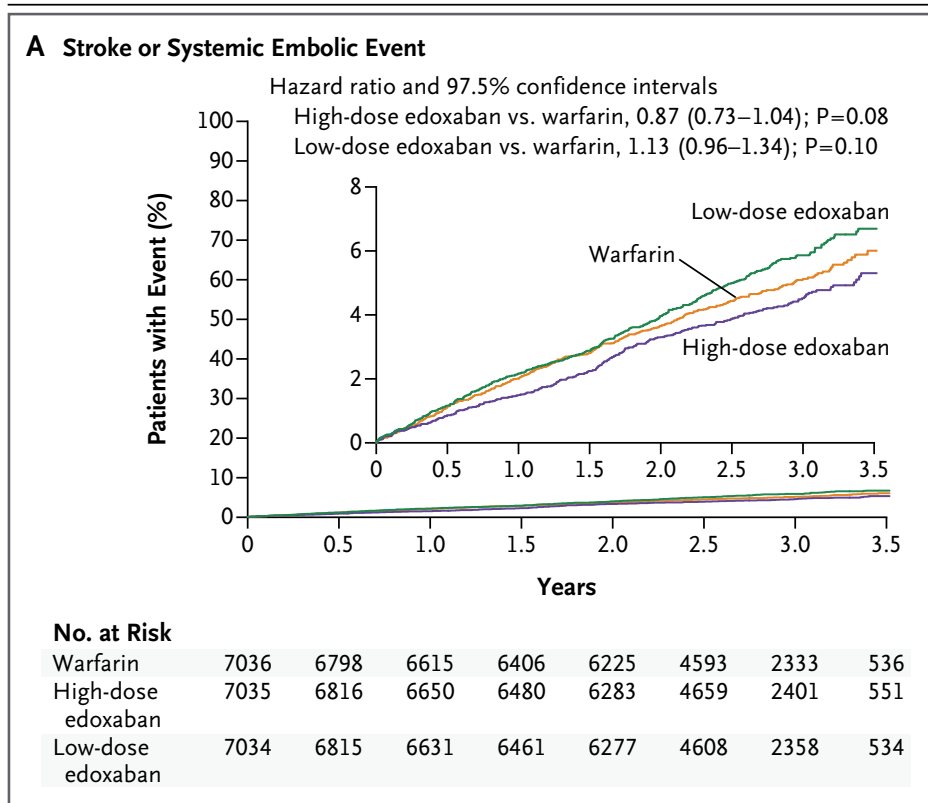

B Major Bleeding

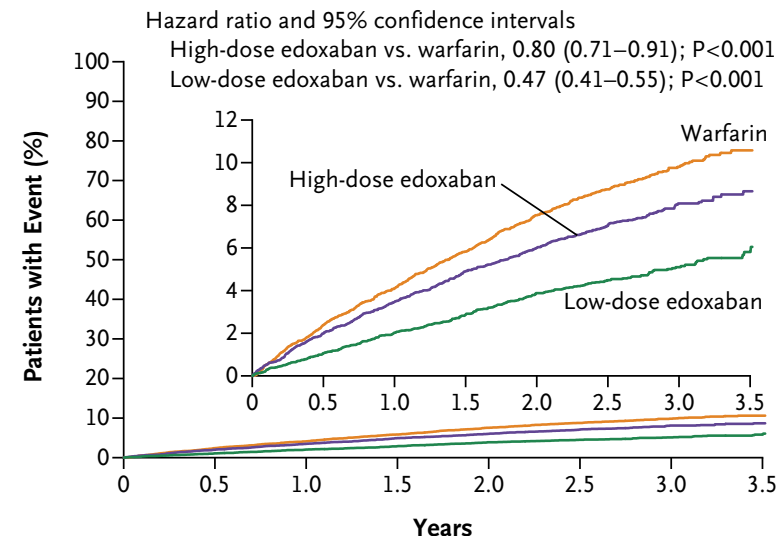

No. at Risk
Warfarin

High-dose

edoxaban

edoxaban

$\begin{array}{llllllll}7012 & 6116 & 5630 & 5278 & 4941 & 3446 & 1687 & 370 \\ 7012 & 6039 & 5594 & 5232 & 4910 & 3471 & 1706 & 345 \\ 7002 & 6218 & 5791 & 5437 & 5110 & 3635 & 1793 & 386\end{array}$

Figure 1. Kaplan-Meier Curves for the Primary Efficacy and Principal Safety End Points.

Panel A shows the cumulative event rates for stroke or systemic embolism in the intention-to-treat population (all patients who underwent randomization) during the overall study period (i.e., beginning from the time of randomization to the end of the double-blind treatment period); data from the overall study period, rather than the treatment period only, were used in the superiority analyses of efficacy. Panel B shows the principal safety outcome of major bleeding, defined according to the criteria of the International Society on Thrombosis and Haemostasis, ${ }^{10}$ in the safety population during the treatment period. The Kaplan-Meier curve was drawn without interval censoring for treatment interruptions. The inset in each panel shows the same data on an enlarged segment of the $y$ axis. (at $\mathrm{P}<0.05$ ) between treatment and subgroups defined according to status with respect to previous receipt of a vitamin $\mathrm{K}$ antagonist (warfarin vs. both edoxaban groups), concurrent aspirin use (warfarin vs. low-dose edoxaban group), and concurrent amiodarone use (warfarin vs. low-dose edoxaban group) (Fig. S3 in the Supplementary Appendix). The reduction in major bleeding with edoxaban as compared with warfarin was significantly greater among patients who received a dose reduction at randomization than among those who did not receive a dose reduction (Fig. $\mathrm{S} 4$ in the Supplementary Appendix).

\section{OTHER SAFETY OUTCOMES}

The rates of adverse events and serious adverse events were similar in the three groups (Table S1 in the Supplementary Appendix). The proportions of patients with an elevated level of liver enzymes or with hepatocellular injury were also similar in the three groups.

\section{COMPARISON OF EDOXABAN REGIMENS}

In the intention-to-treat analysis, the rate of stroke or systemic embolic event was lower with high-dose edoxaban than with low-dose edoxaban $(\mathrm{P}<0.001)$; this difference was driven by a relative reduction in the incidence of ischemic stroke of $29 \%$ with high-dose edoxaban (236 vs. 333 events), which more than offset a higher incidence of hemorrhagic stroke (49 events, vs. 30 events with low-dose edoxaban), although the hemorrhagic strokes had more severe sequelae than the ischemic strokes. As compared with high-dose edoxaban, low-dose edoxaban was associated with significantly lower rates of bleeding, including major bleeding, intracranial bleeding, and major or clinically relevant nonmajor bleeding. There were no significant differences between the two edoxaban groups in the rates of death from cardiovascular causes and death from any cause.

\section{DISCUSSION}

In this trial, both edoxaban regimens were noninferior to well-managed warfarin (median time in the therapeutic range, $68.4 \%$ of the treatment period) for the prevention of stroke or systemic embolic event; the high-dose edoxaban regimen tended to be more effective than warfarin. The rate of ischemic stroke was similar with high- 


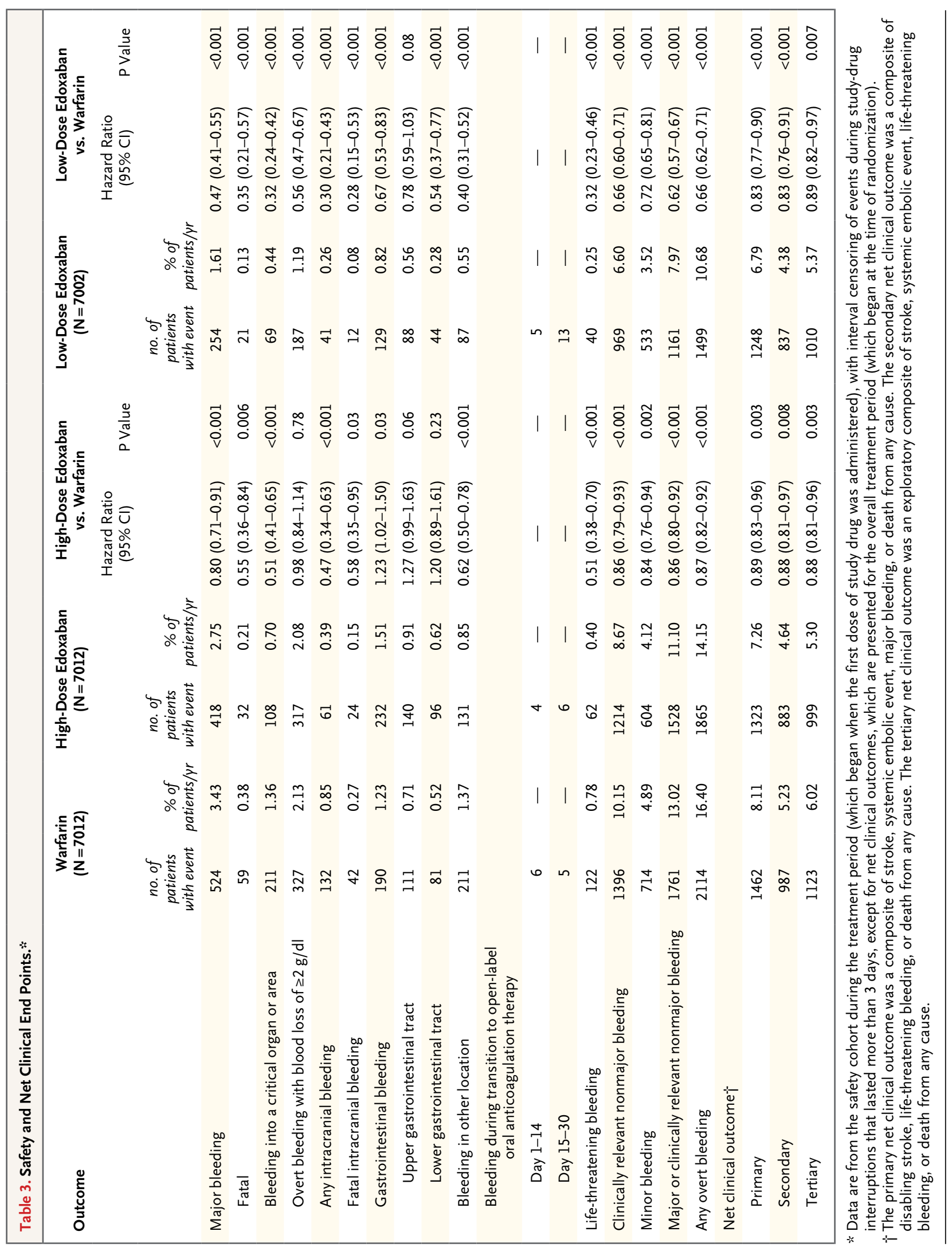


dose edoxaban and warfarin but was higher with the low-dose edoxaban regimen. The incidence of hemorrhagic stroke and the rate of death from cardiovascular causes were significantly lower with both edoxaban regimens than with warfarin.

As compared with warfarin, edoxaban was associated with consistently lower, dose-related rates of all types of bleeding, including major bleeding, intracranial bleeding, and life-threatening bleeding. The single exception was gastrointestinal bleeding, which occurred more frequently with high-dose edoxaban but less frequently with low-dose edoxaban than it did with warfarin. The rates of net clinical outcomes, which were composites of cardiovascular events, death from any cause, or bleeding, were significantly lower with both edoxaban regimens than with warfarin. The very low rate of missing data $(0.5 \%)$ underscores the robustness of these observations. ${ }^{13,14}$

The primary efficacy and safety findings were consistent across major subgroups, including those defined according to demographic characteristics of the patients, risk of stroke (as defined by the $\mathrm{CHADS}_{2}$ score), and geographic region, with three notable exceptions. First, patients who had not previously received a vitamin $\mathrm{K}$ antagonist had significantly fewer stroke or systemic embolic events with high-dose edoxaban than with warfarin, whereas the rates were similar among patients who had previously received a vitamin $\mathrm{K}$ antagonist. Second, the concomitant use of amiodarone and low-dose edoxaban, as well as the concomitant use of aspirin and lowdose edoxaban, appeared to increase the treatment effect of low-dose edoxaban - a finding that was possibly related to a modest increase in edoxaban levels with amiodarone $e^{15}$ and enhanced stroke prevention with aspirin. ${ }^{11}$ Third, a reduction in the edoxaban dose in patients with moderate renal impairment, a body weight of $60 \mathrm{~kg}$ or less, or the concomitant use of P-glycoprotein inhibitors was associated with a decreased risk of bleeding with both regimens. Subgroup analyses exploring relationships among safety, efficacy, and dose reduction (as compared with no dose reduction) showed an even greater reduction in bleeding with edoxaban as compared with warfarin among patients who underwent dose reduction, without an apparent loss in efficacy (Fig. S3 and S4 in the Supplementary Appendix). In addition, dose modifications were permitted after randomization, since factors that affect drug clearance may vary over time.

Edoxaban appeared to be safe, had no unexpected side effects, had fewer side effects than warfarin (as managed with a median time in the therapeutic range of $68.4 \%$ of the treatment period), and had a favorable net clinical outcome. Although no specific antidote for edoxaban is currently available, hemostatic agents reverse its anticoagulant effect. ${ }^{16}$ The availability of a reliable factor Xa assay ${ }^{17}$ and specific reversal strategies $^{18}$ in urgent clinical situations could potentially improve the safety profile of edoxaban, but no particular strategy is well accepted in practice at this time. The rate of myocardial infarction was not altered with edoxaban, and there was no increase in the risk of stroke or bleeding when patients in the edoxaban groups made the transition to open-label anticoagulant therapy at the end of the study.

In previous studies involving patients with atrial fibrillation, dabigatran, rivaroxaban, and apixaban were at least as efficacious as warfarin and were associated with lower rates of intracranial bleeding. ${ }^{19-21}$ Similar to edoxaban, these drugs can be given in fixed doses without routine laboratory monitoring and have fewer drugdrug and food-drug interactions than vitamin $\mathrm{K}$ antagonists. Although there may be subtle differences among the new anticoagulant agents with respect to the prevention of ischemic stroke, myocardial infarction, bleeding, or death, ${ }^{22} \mathrm{di}-$ rect comparative studies are needed to determine whether these are real differences in clinical efficacy and safety or whether they reflect differences in the pharmacologic properties, the doses used, the patient populations, the quality of warfarin management, or other aspects of the trial designs. ${ }^{23}$

Strengths of the ENGAGE AF-TIMI 48 trial include the large sample size, long follow-up, minimal amount of missing data, greater-thanaverage time in the therapeutic range in the warfarin group, and the inclusion of multiple once-daily doses ${ }^{24,25}$ of a new anticoagulant agent ranging from 15 to $60 \mathrm{mg}$ with dynamic dose modification. In addition, the implementation of a comprehensive transition plan to open-label anticoagulation therapy resulted in a low and evenly distributed number of events after the discontinuation of study therapy. This finding 
makes it unlikely that there is a rebound activation of coagulation after the discontinuation of edoxaban.

In conclusion, both once-daily regimens of edoxaban were noninferior to warfarin for the prevention of stroke or systemic embolism and were associated with significantly lower rates of bleeding and death from cardiovascular causes.

Supported by Daiichi Sankyo Pharma Development.

Dr. Giugliano reports receiving consulting fees from Daiichi Sankyo, Janssen Pharmaceuticals, and Merck; lecture fees from Bristol-Myers Squibb, Daiichi Sankyo, Merck, and Sanofi; and grant support through his institution from Daiichi Sankyo, Merck, Johnson \& Johnson, Sanofi, and AstraZeneca. Dr. Ruff reports receiving consulting fees from Daiichi Sankyo, Bristol-Myers Squibb, and Boehringer Ingelheim and grant support through his institution from Daiichi Sankyo. Dr. Braunwald reports receiving consulting fees from Sanofi, Genzyme, Amorcyte, the Medicines Company, and Cardiorentis; lecture fees from Eli Lilly, Menarini, Medscape, and Bayer HealthCare; and grant support through his institution from Daiichi Sankyo, AstraZeneca, Johnson \& Johnson, GlaxoSmithKline, Bristol-Myers Squibb, Beckman Coulter, Roche Diagnostics, Pfizer, Merck, and Sanofi. He also reports serving as an unpaid consultant for Merck and providing uncompensated lectures for Merck and CVRx. Dr. Wiviott reports receiving consulting fees from AstraZeneca, Bristol-Myers Squibb, Eisai, Arena Pharmaceuticals, Eli Lilly, Daiichi Sankyo, Aegerion, AngelMed, Janssen Pharmaceuticals, Xoma, ICON Clinical Research, and Boston Clinical Research Institute and grant sup- port through his institution from AstraZeneca, Bristol-Myers Squibb, Eisai, Arena Pharmaceuticals, Merck, Eli Lilly, Daiichi Sankyo, and Sanofi. Dr. Halperin reports receiving consulting fees from Bayer HealthCare, Boehringer Ingelheim, Johnson \& Johnson, Ortho-McNeil-Janssen Pharmaceuticals, Pfizer, Sanofi, Biotronik, Boston Scientific, and Medtronic. Dr. Waldo reports receiving consulting fees from Daiichi Sankyo, Sanofi, CardioInsight, Pfizer, and ChanRx; lecture fees from Sanofi, Pfizer, Janssen Pharmaceuticals, and Bristol-Myers Squibb; honoraria for clinical trial-related activities from Biotronik, St. Jude Medical, Daiichi Sankyo, Biosense Webster, Gilead, and AtriCure; and grant support through his institution from Gilead. Dr. Ezekowitz reports receiving consulting fees from Boehringer Ingelheim, Pfizer, Sanofi, Bristol-Myers Squibb, Portola Pharmaceuticals, Bayer HealthCare, Daiichi Sankyo, Medtronic, Aegerion, Merck, Johnson \& Johnson, Gilead, Janssen Scientific Affairs, Pozen, and Coherex Medical; and lecture fees from Boehringer Ingelheim. Dr. Weitz reports receiving consulting fees from Boehringer Ingelheim, Bayer HealthCare, Bristol-Myers Squibb, Daiichi Sankyo, Janssen Pharmaceuticals, Pfizer, Merck, and Portola Pharmaceuticals. Dr. Koretsune reports receiving lecture fees from Daiichi Sankyo, Boehringer Ingelheim, Pfizer, Bayer HealthCare, and Bristol-Myers Squibb; and grant support through his institution from Daiichi Sankyo and Boehringer Ingelheim. Drs. Shi, Patel, Hanyok, and Mercuri report being employees of Daiichi Sankyo. Dr. Mercuri also reports holding a pending patent related to the clinical properties of edoxaban. Ms. Murphy, Ms. Grip, and Dr. Antman report receiving grant support through their institution from Daiichi Sankyo. No other potential conflict of interest relevant to this article was reported.

Disclosure forms provided by the authors are available with the full text of this article at NEJM.org.

\section{REFERENCES}

1. Matsushima N, Lee F, Sato T, Weiss D, Mendell J. Bioavailability and safety of the factor Xa inhibitor edoxaban and the effects of quinidine in healthy subjects. Clin Pharm Drug Dev 2013;2:358-66.

2. Ogata $\mathrm{K}$, Mendell-Harary J, Tachibana $\mathrm{M}$, et al. Clinical safety, tolerability, pharmacokinetics, and pharmacodynamics of the novel factor Xa inhibitor edoxaban in healthy volunteers. J Clin Pharmacol 2010; 50:743-53.

3. Weitz JI, Connolly SJ, Patel I, et al. Randomised, parallel-group, multicentre, multinational phase 2 study comparing edoxaban, an oral factor $\mathrm{Xa}$ inhibitor with warfarin for stroke prevention in patients with atrial fibrillation. Thromb Haemost 2010;104:633-41.

4. Salazar DE, Mendell J, Kastrissios H, et al. Modelling and simulation of edoxaban exposure and response relationships in patients with atrial fibrillation. Thromb Haemost 2012;107:925-36.

5. The Hokusai-VTE Investigators. Edoxaban versus warfarin for the treatment of symptomatic venous thromboembolism. N Engl J Med 2013;369:1406-15.

6. Ruff CT, Giugliano RP, Antman EM, et al. Evaluation of the novel factor $\mathrm{Xa}$ in hibitor edoxaban compared with warfarin in patients with atrial fibrillation: design and rationale for the Effective aNticoaGulation with factor xA next GEneration in
Atrial Fibrillation-Thrombolysis In Myocardial Infarction study 48 (ENGAGE AF-TIMI 48). Am Heart J 2010;160:635-41.

7. Gage BF, Waterman AD, Shannon $W$, Boechler M, Rich MW, Radford MJ. Validation of clinical classification schemes for predicting stroke: results from the National Registry of Atrial Fibrillation. JAMA 2001;285:2864-70.

8. Rosendaal FR, Cannegieter SC, van der Meer FJ, Briët E. A method to determine the optimal intensity of oral anticoagulant therapy. Thromb Haemost 1993;69:236-9. 9. Verhovsek M, Motlagh B, Crowther MA, et al. Quality of anticoagulation and use of warfarin-interacting medications in long-term care: a chart review. BMC Geriatr 2008;8:13.

10. Schulman S, Kearon C. Definition of major bleeding in clinical investigations of antihemostatic medicinal products in non-surgical patients. J Thromb Haemost 2005;3:692-4.

11. Hart RG, Pearce LA, Aguilar MI. Meta-analysis: antithrombotic therapy to prevent stroke in patients who have nonvalvular atrial fibrillation. Ann Intern Med 2007;146:857-67.

12. Food and Drug Administration. Guidance for industry: non-inferiority clinical trials. 2010 (http://www.fda.gov/downloads/ drugs/guidancecomplianceregulatory information/guidances/ucm202140.pdf).
13. Little RJ, D’Agostino R, Cohen ML, et al. The prevention and treatment of missing data in clinical trials. $\mathrm{N}$ Engl J Med 2012;367:1355-60.

14. Ware JH, Harrington D, Hunter DJ, D’Agostino RB. Missing data. N Engl J Med 2012;367:1353-4.

15. Mendell J, Zahir H, Matsushima N, et al. Drug-drug interaction studies of cardiovascular drugs involving P-glycoprotein, an efflux transporter, on the pharmacokinetics of edoxaban, an oral factor Xa inhibitor. Am J Cardiovasc Drugs 2013; 13:331-42.

16. Fukuda T, Honda Y, Kamisato C, Morishima Y, Shibano T. Reversal of anticoagulant effects of edoxaban, an oral, direct factor Xa inhibitor, with haemostatic agents. Thromb Haemost 2012;107: 253-9.

17. Samama MM, Mendell J, Guinet C, Le Flem L, Kunitada S. In vitro study of the anticoagulant effects of edoxaban and its effect on thrombin generation in comparison to fondaparinux. Thromb Res 2012;129(4):e77-e82.

18. Laulicht B, Bakhru S, Jiang X, et al. Antidote for new oral anticoagulants: mechanism of action and binding specificity of PER977. Presented at the XXIV Congress of the International Society on Thrombosis and Haemostasis, Amsterdam, June 29-July 4, 2013. abstract. 
19. Connolly SJ, Ezekowitz MD, Yusuf S, lar atrial fibrillation. N Engl J Med 2011; et al. Dabigatran versus warfarin in pa- 365:883-91.

tients with atrial fibrillation. N Engl J Med 22. Dogliotti A, Paolasso E, Giugliano RP. 2009;361:1139-51. [Erratum, N Engl J Med Novel oral anticoagulants in atrial fibril2010;363:1877.]

20. Granger $\mathrm{CB}$, Alexander JH, McMurray $\mathrm{JJV}$, et al. Apixaban versus warfarin in patients with atrial fibrillation. $\mathrm{N}$ Engl J Med 2011;365:981-92.

21. Patel MR, Mahaffey KW, Garg J, et al. Rivaroxaban versus warfarin in nonvalvu- lation: a meta-analysis of large, randomized, controlled trials vs warfarin. Clin Cardiol 2013;36:61-7.

23. Grip LT, Ruff CT, Giugliano RP. New oral antithrombotic strategies: 2013 update on atrial fibrillation. Hot Topics Cardiol 2013;31:7-18.
24. Renda G, De Caterina R. The new oral anticoagulants in atrial fibrillation: once daily or twice daily? Vascul Pharmacol 2013;59:53-62.

25. Laliberté F, Nelson WW, Lefebvre P, Schein JR, Rondeau-Leclaire J, Duh MS. Impact of daily dosing frequency on adherence to chronic medications among nonvalvular atrial fibrillation patients. Adv Ther 2012;29:675-90.

Copyright @) 2013 Massachusetts Medical Society.

AN NEJM APP FOR IPHONE

The NEJM Image Challenge app brings a popular online feature to the smartphone. Optimized for viewing on the iPhone and iPod Touch, the Image Challenge app lets you test your diagnostic skills anytime, anywhere. The Image Challenge app randomly selects from 300 challenging clinical photos published in NEJM, with a new image added each week. View an image, choose your answer, get immediate feedback, and see how others answered.

The Image Challenge app is available at the iTunes App Store. 